\title{
DIFFRACTIVE PRODUCTION OF VECTOR MESONS IN LEPTON-NUCLEON REACTIONS *
}

\author{
M.-S. CHEN, F.S. HENYEY ${ }^{\star}$ and G.L. KANE \\ Randall Laboratory of Physics, University of Michigan, Ann Arbor MI 48109
}

Received 13 July 1976

\begin{abstract}
We calculate cross sections for the production of vector mesons $\mathrm{V}$ in reactions $Q+$ $\mathrm{N} \rightarrow \ell^{\prime}+\mathrm{V}+\mathrm{N}$, with $\ell, \ell^{\prime}=\nu$, e, $\mu$, taking into account all presently known information from electroproduction of $\rho$ 's. This leads to considerable differences in results from previous calculations, often more than an order of magnitude. The results for the $\rho$ are then largely a translation of electroproduction data into $v$ predictions and they will provide a direct test of the relation of weak and electromagnetic currents. The $y$ or $Q^{2}$ distributions for the $\rho$ are instructive. The prediction for the $A_{1}$ provides a direct test of the existence of the $A_{1}$ and of whether vector and axial vector currents materialize into particles in the same way. The detection of a $B$ meson would provide direct evidence for second-class currents and a measurement of their strength. We estimate all $F^{*}$ production will account for at most $2 \%$ of the total $\nu$ cross section at FNAL energies, which must be multiplied by the muon semi-leptonic branching ratio (and possibly a factor of 2 for an axial vector $\mathrm{F}^{*}$ ) to calculate its contribution to the dimuon event rate.
\end{abstract}

\section{Introduction}

One way which may be very fruitful to probe the structure of weak and electromagnetic (charged and neutral) currents is to study how the currents convert diffractively into particle states. For real photons (i.e., for the electromagnetic current at $Q^{2}=0$ ) the answer is known and at least descriptively understood:

$$
\gamma \mathrm{p} \rightarrow \rho^{0} \mathrm{p}, \omega^{0} \mathrm{p}, \varphi \mathrm{p}, \psi \mathrm{p}, \psi^{\prime} \mathrm{p}
$$

have been observed and the coupling of $\psi, \psi^{\prime}$ to the electromagnetic current have been measured.

For the virtual electromagnetic current in electroproduction $\left(Q^{2} \geqslant 0\right), \rho$ and $\omega$ production have also been observed and studied and information gained about the $Q^{2}$ dependence of the dynamics.

\footnotetext{
* Research supported in part by the US Energy Research and Development Agency.

$\star \star$ Present address: Department of Physics, University of California San Diego, La Jolla

CA 92037.
} 
In general for the weak and electromagnetic currents $J$ in the leptoproduction reactions

$$
\mathrm{e}^{-} \mathrm{p} \rightarrow \mathrm{e}^{-} \rho^{0} \mathrm{p}, \quad \nu \mathrm{p} \rightarrow \mu^{-} \rho^{+} \mathrm{p}, \quad \nu \mathrm{p} \rightarrow \nu \rho^{0} \mathrm{p}, \quad \text { etc. }
$$

one is studying the matrix element $\langle V|J| 0\rangle$ for each vector meson into which the current can convert. One directly learns about the quantum numbers of the currents, about their coupling strenghs, and by studying the dependence on kinematical variables, about their space-time structure.

One can look for many vector mesons in such processes, old particles and new ones. In this note we will concentrate on a few $\left(\rho^{+}, \rho^{0}, \mathrm{~A}_{1}^{+}, \mathrm{A}_{1}^{0}, \mathrm{~B}^{+}, \mathrm{B}^{0}, \mathrm{~F}^{*+}\right)$ because they are the ones most likely to be studied in experiments and their presence or absence has immediate important consequences. We will present the calculational procedure so that anyone can easily (with some numerical effort) work out the results for other mesons.

Let us discuss briefly the physics expected from each of these states. Anyone unfamiliar with the kinematical variables can find them defined below. The electroproduction of $\rho$ has been studied in detail $[1,2]$ for SLAC and Cornell energies and $Q^{2}<2 \mathrm{GeV}^{2}$. Consequently, for $\nu \mathrm{p} \rightarrow \mu^{-} \rho^{+} \mathrm{p}$ the cross section $\mathrm{d} \sigma / \mathrm{d} Q^{2} \mathrm{~d} \nu$ is directly given by the basic hypothesis that the nonstrange weak charged current and the electromagnetic current are in the same isospin multiplet. The total rate, i.e., the cross section integrated over $Q^{2}$ and $v$ (or $x$ and $y$ ), depends a little on extra. polations outside the region where it is measured in electroproduction, but the total rate is probably predictable to better than a factor of two. It is important that these events be found experimentally. The $Q^{2}$ or $y$ distributions for the $\rho^{+}$will directly provide new information on $\langle V|J| 0\rangle$ at larger $Q^{2}$; the $x$ distribution is dominated by phase space and $t_{\min }$ effects, and provides somewhat less interesting information for low statistics experiments.

The production of $\rho^{0}$ is due to an isovector neutral vector current. If the dynamics of charged and neutral currents were alike there would just be a proportionality factor $\frac{1}{2}\left(1-2 \sin ^{2} \theta_{W}\right)^{2}$ between $\rho^{+}$and $\rho^{0}$, so the relative rate is a measure of $\sin ^{2} \theta_{\mathrm{W}}$. If $\sin ^{2} \theta_{\mathrm{W}} \sim 0.4$ as presently [3] believed, $\rho^{0}$ production is suppressed by a factor of about 50 , so it should not be seen for some time.

Next consider the $A_{1}$, the isovector meson which is supposed to play the same role for the axial vector current that the $\rho$ does for the vector current. So far it has not been possible to find an $A_{1}$ experimentally and there is reason to believe [4] that existing non-diffractive experiments in hadron reactions are more than sensitive enough to have seen an $A_{1}$ if there was one. It is already possible to argue [5] that existing information implies that the ways vector and axial vector currents materialize into particles is very asymmetric. But the crucial test will come in the reactions under consideration here, such as $\nu \mathrm{p} \rightarrow \mu^{-} \mathrm{A}_{1}^{+} \mathrm{p}$, where the weak charged axial vector current can directly materialize into an $\mathrm{A}_{1}$ just as the electromagnetic and weak vector currents into a $\rho$. Our predictions for the $A_{1}^{+}$rate must be a little less firm 
than for the $\rho$ as the axial current is involved and there is a change in mass scale, but they should be serious at about the factor of 2-3 level. If the indications from hadronic reactions that there is no $A_{1}$ are confirmed here it would be of considerable significance for both weak interaction theory and for quark models.

For the neutral axial vector current there is no suppression as in the vector case so $\nu \mathrm{p} \rightarrow \nu \mathrm{A}_{1}^{0} \mathrm{p}$ should occur, with $\sigma\left(\mathrm{A}_{1}^{0}\right) \approx \sigma\left(\mathrm{A}_{1}^{+}\right) \sigma(\mathrm{NC}) / \sigma(\mathrm{CC})$.

If there are charged second class currents they may have the quantum numbers of the $\mathrm{B}$ meson, and diffractive production of the $\mathrm{B}^{+}$may be the most direct test of their presence in particle physics [6]. In one model [6], the rates are predicted to be large with $\mathrm{B}^{+}$production comparable to $\rho^{+}$for $E_{\nu} \sim 5-10 \mathrm{GeV} / c$, and larger at higher energies. Because of the hemiticity properties of second class currents, the $\mathbf{B}^{0}$ is not expected to couple to a neutral second class current in the model of ref. [6], but it would in the model of ref. [7] which introduces an extra neutrino.

A number of people $[8,9]$ have considered production of $F^{*+}$ to see if its semileptonic decay to muons could account for the dimuon events observed at Fermilab. They have used different assumptions and the answers have differed considerably. Since we are careful to use procedures which give agreement with electroproduction data including the $Q^{2}$ dependence for the $\rho$, it is only in the scaling to the $\mathrm{F}^{*}$ mass and quantum numbers that we could go very wrong. By resolving all ambiguous choices to obtain as large a cross section as we can, we find that we can only get a total $\mathrm{F}^{*}$ production, $\nu+\mathrm{p} \rightarrow \mu^{-} \mathrm{F}^{*+}+$ anything, of about $2 \%$ of $\sigma_{\mathrm{T}}(\nu \mathrm{p})$. Since this must be reduced by the semileptonic branching ratio to give a muon, and possibly enhanced by a factor of two for an axial vector $F^{*}$, it can only account for a fraction but perhaps not a small fraction of the observed dimuons. For $\bar{\nu}$ reactions the $\mathrm{F}^{*}$ rate will be approximately the same so it will account for a 3 times larger part of $\sigma_{\mathrm{T}}(\bar{\nu})$, perhaps almost $1 \%$.

Over the years, a number [8-11] of authors have carried out calculations such as ours. The reason we have produced yet another one is that it turns out that the results are more sensitive to the assumptions on $Q^{2}$ dependence than people expected, and also that the observed $Q^{2}$ dependence in electroproduction is different from what was expected. Previously published estimates vary by more than an order of magnitude from ours in both directions. It does not seem fruitful to point out in each case where we differ from other authors, and partially because of the strong dependence on the kinematics and on approximations in doing phase space integrations we have not been able to locate the source of all discrepancies. We will explain in detail why we proceed as we do and the consequences of other choices, and this should suffice to enable the reader to understand what is happening.

In the next section we give the standard cross section formula and define variables and cross sections. In sect. 3 we discuss the $Q^{2}$ dependence of the transverse cross section, and in sect. 4 the $Q^{2}$ dependence of the longitudinal cross section. In sect. 5 we comment on the kinematics and on integration limits. Finally in sect. 6 we present the results for expected rates for all the mesons and for some detailed distributions for the $\rho$. 


\section{Definitions and cross-section formula}

Let the initial and final leptons have momenta $q$ and $q^{\prime}$, and the initial and final nucleons $p$ and $p^{\prime}$. Then $\left(p^{2}=p^{\prime 2}=-m_{\mathrm{N}}^{2}\right)$

$$
\begin{aligned}
& Q^{2}=\left(q-q^{\prime}\right)^{2} \gtrsim 0, \\
& t=-\left(p^{\prime}-p\right)^{2} \lesssim 0,
\end{aligned}
$$

$E$ is the lab beam energy,

$E^{\prime}$ is the final lepton energy,

$y=\left(E-E^{\prime}\right) / E$,

$x=Q^{2} / 2 m_{\mathrm{N}} E y$,

$W^{2}=$ hadron energy squared $=m_{\mathrm{N}}^{2}+2 m_{\mathrm{N}} E y-Q^{2}$.

Fig. 1 defines some of the kinematics.

The standard cross-section formula is

$$
\begin{gathered}
\frac{\mathrm{d} \sigma}{\mathrm{d} t \mathrm{~d} x \mathrm{~d} y}=\frac{G_{\mathrm{f}}^{2} M_{\mathrm{N}} E \cos ^{2} \theta}{2 \pi^{2}} \frac{1}{\gamma_{\mathrm{V}}^{2}} Q^{2}(1-x)\left\{y^{2} \frac{\mathrm{d} \sigma_{\perp}}{\mathrm{d} t}(x, y, t)\right. \\
\left.+\frac{2\left(1-y-M_{\mathrm{N}} x y / 2 E\right)}{1+2 x y M_{\mathrm{N}} / E}\left(\frac{\mathrm{d} \sigma_{\perp}}{\mathrm{d} t}(x, y, t)+\frac{\mathrm{d} \sigma_{\mathrm{L}}}{\mathrm{d} t}(x, y, t)\right)\right\},
\end{gathered}
$$

where $\theta_{\mathrm{C}}$ is the Cabibbo angle, $\cos ^{2} \theta_{\mathrm{C}} \simeq 0.95, \gamma_{\mathrm{V}}$ measures the current-meson coupling, $\mathrm{d} \sigma_{\perp} / \mathrm{d} t$ and $\mathrm{d} \sigma_{\mathrm{L}} / \mathrm{d} t$ are the transverse and longitudinal cross sections for $\gamma+\mathrm{N} \rightarrow \mathrm{V}+\mathrm{N}$ for a virtual photon. We assume these cross sections depend on $t$ approximately exponentially; this is consistent with the electroproduction $[1,2]$ data. Then the integral over $t$ is aut omatic and

$$
\int \mathrm{d} t \frac{\mathrm{d} \sigma}{\mathrm{d} t}(x, y, t) \rightarrow \sigma(x, y) \mathrm{e}^{B t_{\min }} .
$$

The forms of $\sigma_{\perp}$ and $\sigma_{\mathrm{L}}$ will be specified in the next two sections. The vector-

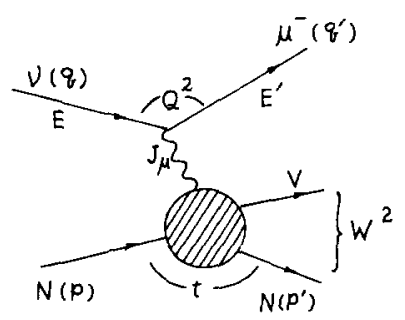

Fig. 1. Kinematics for the diffractive production of vector mesons in neutrino-nucleon reactions. 
dominance factor $M_{\mathrm{V}}^{4} /\left(M_{\mathrm{V}}^{2}+Q^{2}\right)^{2}$ is included in $\sigma_{\perp}$ rather than exhibited directly in $\mathrm{d} \sigma / \mathrm{d} x \mathrm{~d} y$ for reasons discussed below.

The current-meson coupling is fixed as follows. For $\rho^{+}$it is the standard vector domance result $\gamma_{\rho}^{2} / 4 \pi \simeq 2.6$. For $\rho^{0}$ it is the isovector neutral current coupling to $\rho$, which has a factor $\frac{1}{2}\left(1-2 \sin ^{2} \theta_{W}\right)^{2}$ in $1 / \gamma^{2}$ in the Weinberg-Salam theory. For $\mathrm{A}_{1}$, we assume the expected result form the Weinberg sum rules, $f_{\rho}=f_{\mathrm{Al}}$ and $m_{\rho}^{2}=\frac{1}{2} m_{\mathrm{Al}}^{2}$. Then with $f_{\mathrm{V}}=m_{\mathrm{V}}^{2} / \gamma_{\mathrm{V}}$ we have $\gamma_{\mathrm{A} 1}^{2}=4 \gamma_{\rho}^{2}$, for the charged currents. For the $\mathrm{B}^{+}$we have suggested elsewhere [6] that $\left|f_{\mathrm{B}}\right| \stackrel{\rho}{\simeq} 3\left|f_{\rho}\right|$ so $\gamma_{\mathrm{B}}^{2} \simeq 0.6 \gamma_{\rho}^{2}$. For $\mathrm{F}^{*}$ we use the $\mathrm{SU}(4)$ relations and we assume from the neutral particles that the quantity $m_{\mathrm{V}} / \gamma_{\mathrm{V}}^{2}$ is symmetric, so $\gamma_{\mathrm{F}}^{2} \simeq m_{\mathrm{F}^{*}} \gamma_{\rho}^{2} / 2 m_{\mathrm{p}} \simeq 1.5 \gamma_{\rho}^{2}$.

Finally we note that the $Q^{2}$ factor in eq. (2), which is important numerically in suppressing the entire diffractive contribution, is quite general, coming effectively from the sum over lepton spins giving a factor $p_{\nu} \cdot p_{\mu}$ which is $Q^{2}$ when the muon mass is neglected.

\section{3. $\underline{Q}^{2}$ Dependence of $\sigma_{\downarrow}$}

The conventional vector-dominance treatment of $\sigma_{\perp}$ is to assume that it has the $Q^{2}$ dependence of $\sigma_{\text {tot }}$, from the $\rho$ propagator,

$$
\sigma_{\perp} \rightarrow\left(M_{\mathrm{V}}^{2} /\left(M_{\mathrm{V}}^{2}+Q^{2}\right)\right)^{2} \sigma_{\perp}^{\prime}
$$

and then $\sigma_{\perp}^{\prime}$ is taken to be constant at the $Q^{2}=0$ value. However, this is known experimentally to be wrong. For example, in fig. 2 we reproduce the data from ref. [2]. The ratio $o_{\rho} / v_{\text {tot }} \equiv v(\gamma \mathrm{N} \rightarrow \rho \mathrm{N}) / o_{\text {tot }}(\gamma \mathrm{N})$ falls about a factor of three as $Q^{2}$ increases from 0 to about $0.5 \mathrm{GeV}^{2}$, and then levels out or falls more slowly. Consequently, over much of the $Q^{2}$ range the cross sections which should be used in

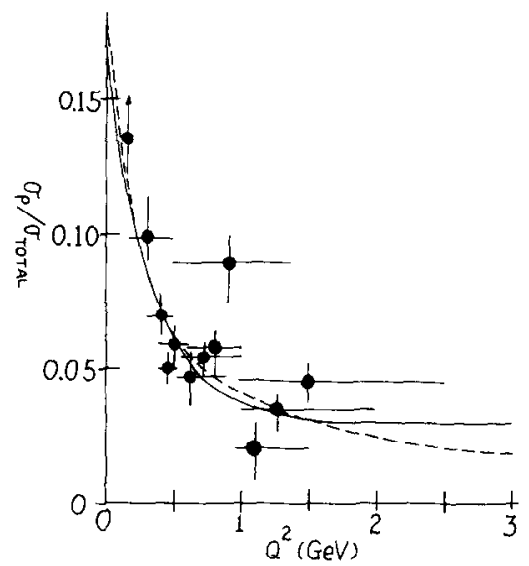

Fig. 2. The $Q^{2}$ dependence of $\sigma_{\rho} / \sigma_{\text {tot }}$ in electroproduction, where the data are taken from ref. [2], the solid line represents the fit by $F_{2}\left(Q^{2}\right)$, and the dashed line represents the fit by $F_{1}\left(Q^{2}\right)$. 
eq. 1 are about three times smaller than at $Q^{2}=0$, so that predicted $\mathrm{d} \sigma / \mathrm{d} x \mathrm{~d} y$ are correspondingly smaller. At larger $Q^{2}$ the effect may be much more important. Since $\nu$ reactions do not have a propogator associated with the current the integrations to get the total rate go to much large $Q^{2}$ than in electroproduction, and predicted total rates can be much larger than they should be if this $Q^{2}$ fall-off is not included.

Since electroproduction data is only available for $Q^{2} \lesssim 2 \mathrm{GeV}^{2}$, we proceed as follows. First we note [12] that $\sigma_{\text {tot }}\left(\right.$ i.e., $\nu W_{2}$ ) from electroproduction is better described by the function $F_{1}\left(Q^{2}\right)$,

$$
\sigma_{\text {tot }}=\frac{0.36}{0.36+Q^{2}} \sigma \equiv F_{1}\left(Q^{2}\right) \sigma,
$$

(where $\sigma$ is for normalization) which falls slightly less fast than the simple form of eq. (3). The vector-dominance factor is already absorbed in the definition of $F_{1}$, and is not to be included in addition. Next we choose a paramaterization of the data of fig. 2 which goes to a constant for large $Q^{2}$,

$$
\begin{aligned}
& F_{2}\left(Q^{2}\right)=\left(0.04+0.13 \mathrm{e}^{-3 Q^{2}}\right) / 0.17, \\
& \sigma_{\rho} / \sigma_{\text {tot }} \approx 0.17 F_{2}\left(Q^{2}\right) \approx 0.17 F_{1}\left(Q^{2}\right) .
\end{aligned}
$$

Eqs. (4)-(6) define $F_{1}$ and $F_{2}$, both normalized to unity at $Q^{2}=0$. Note that both $F_{1}$ and $F_{2}$ give adequate descriptions of $\sigma_{\rho} / \sigma_{\text {tot }}$ where it is measured, while $F_{1}$ describes $\sigma_{\text {tot }}$.

Now, for the $\rho^{+}$, we calculate with the following choices:

$$
\text { (i) } \sigma_{\perp}=F_{1}\left(Q^{2}\right) F_{2}\left(Q^{2}\right) \frac{\sigma_{0}^{2}}{16 \pi B} \frac{1}{0.389} \mathrm{e}^{B t_{\min }}
$$

This can be interpreted as taking $\sigma_{\text {tot }}$ from the electroproduction fit plus $\sigma_{\rho} / \sigma_{\text {tot }}$ from fig. 2, normalizing to the $Q^{2}=0$ value. Neglecting the $W^{2}$ dependence, $\sigma_{0}$ is given by $25 \mathrm{mb}$ for the $\rho$ (and also for $\mathrm{A}_{1}$ and $\mathrm{B}, 10 \mathrm{mb}$ for $\mathrm{F}^{*}$ ), the slope $B$ is 7 $\mathrm{GeV}^{-2}$ for $\rho, \mathrm{A}_{1}$, and $\mathrm{B}$ and $5 \mathrm{GeV}^{-5}$ for $\mathrm{F}^{*}$, and $1 / 0.389$ allows both factors of $\sigma_{0}$ to be in mb. $F_{1}$ includes the vector dominance factor $M_{\mathrm{V}}^{2} /\left(M_{\mathrm{V}}^{2}+Q^{2}\right)^{2}$ in its parametrization.

(ii) As in (i) but replace $F_{1}\left(Q^{2}\right)$ by the vector dominance expectation,

$$
F_{1}\left(Q^{2}\right) \rightarrow\left(M_{\rho}^{2} /\left(M_{\rho}^{2}+Q^{2}\right)\right)^{2} .
$$

For $Q^{2} \lesssim 1 \mathrm{GeV}^{2}$ these are not very different, but eq. (8) falls a power of $Q^{2}$ faster so they give completely different $y$ distributions and different rates.

(iii) As in (i) but replace $F_{2}$ by the assumption that $\sigma_{\rho} / \sigma_{\text {tot }}$ keeps dropping with increasing $Q^{2}$ rather than levelling off,

$$
F_{2}\left(Q^{2}\right) \rightarrow F_{1}\left(Q^{2}\right) \text {. }
$$


Again, this has an extra power of $1 / Q^{2}$ for large $Q^{2}$ compared to eq. (7) so it gives results more like (ii) for the $y$ distributions.

While alternative (i) may be slightly preferable intuitively, we have no solid basis for deciding among these so we calculate with all of them for the $\rho$. They give quite different $y$ distributions at large $y$ but only at high energies do they give significantly different total rates. For the other particles we use (i), which gives the largest rates, and (iii) which gives almost the smallest rates compatible with the electroproduction data, in the $10-20 \mathrm{GeV}$ region. This is preferable for $\mathrm{F}^{*}$, but not necessarily for $\mathrm{A}_{1}$ and $B$ since different physics questions are involved.

We will compare cases (i)-(iii) with case (iv):

$$
\sigma_{\perp}=\left(\frac{M_{\rho}^{2}}{M_{\rho}^{2}+Q^{2}}\right)^{2} \frac{\sigma_{0}^{2}}{16 \pi} \frac{1}{0.389} \mathrm{e}^{B t_{\min }}
$$

which is a naive vector-dominance model form which disagrees considerably with the electroproduction data as discussed above. If there were no electroproduction data we would have used this form.

As we go from the $\rho$, where there is electroproduction data, to the other particles we must decide how to scale the parameters $M_{\rho}^{2}$ in eq. (8) and 0.36 in eq. (4). From vector dominance we certainly expect that both scale as $M_{\mathrm{V}}^{2}$ for a vector meson $\mathrm{V}$, so we use the appropriate $M_{\mathrm{V}}^{2}$ in eq. (8) and we replace

$$
0.36 \rightarrow 0.36 M_{\mathrm{V}}^{2} / M_{\rho}^{2} \text {. }
$$

To see the effect of this, imagine integrating over $Q^{2}$. Then

$$
\sigma \propto Q^{2} \mathrm{~d} Q^{2} \frac{0.36}{0.36+Q^{2}}
$$

so after scaling the parameter

$$
\begin{aligned}
\sigma \propto & \frac{Q^{2} \mathrm{~d} Q^{2} 0.36 M_{\mathrm{V}}^{2} / M_{\rho}^{2}}{0.36 M_{\mathrm{V}}^{2} / M_{\rho}^{2}+Q^{2}} \\
& =\frac{M_{\mathrm{V}}^{4}}{M_{\rho}^{4}}\left(\frac{Q^{2}}{M_{\mathrm{V}}^{2} / M_{\rho}^{2}}\right) \mathrm{d}\left(\frac{Q^{2}}{M_{\mathrm{V}}^{2} / M_{\rho}^{2}}\right) \frac{0.36}{0.36+Q^{2} /\left(M_{\mathrm{V}}^{2} / M_{\rho}^{2}\right)}
\end{aligned}
$$

so, if we were in the high-energy limit and could scale in $Q^{2}$ without kinematical effects, such a procedure would increase the cross section from a heavier vector meson by $M_{\mathrm{V}}^{4} / M_{\rho}^{4}$ because it reduces the fall-off rate in $Q^{2}$. For the $\mathrm{F}^{*}$ this gives a big factor, and for the $A_{1}$ it cancels the suppression by $M^{4}$ in $\gamma_{\mathrm{V}}^{2}$. The full effect is not realized at typical experimental energies because of kinematical cut-off effects. If anyone has any reason to argue that such scaling does not occur, then they will predict smaller cross sections for heavier vector mesons. 
To conclude this section we remark that the fall-off with $Q^{2}$ of $\sigma_{\perp} / \sigma_{\text {tot }}$ has important phenomenological consequences and must be included, even though its origin may not be well understood. It is a violation of naive vector dominance since there both $\sigma_{\perp}$ and $\sigma_{\text {tot }}$ only have the $\rho$ propagator squared. It would be wrong to imagine that the optical theorem gave $\sigma_{\perp} \propto \sigma_{\text {tot }}^{2} / B$ to "explain" the observed fall of $\sigma_{\perp} / \sigma_{\text {tot }}$, since this optical theorem would require two currents,

$$
J N \rightarrow J N, \quad \text { rather than just one } J N \rightarrow \rho N .
$$

It is not clear whether a more sophisticated vector dominance model (for examiple, involving the $\rho^{\prime}$ as well as the $\rho$ ) can simultaneously describe the behaviors of $\sigma_{\text {tot }}$ and $\sigma_{\perp} / \sigma_{\text {tot }}$. In any case, for our purposes in this paper only the fact that the falloff occurs in the data is relevant and we use the data (in parametrized form) directly.

\section{4. $Q^{2}$ dependence of $\sigma_{L}$}

We define as usual $R=\sigma_{\mathrm{L}} / \sigma_{\perp}$. In the past little was known about $\sigma_{\mathrm{L}}$ or $R$, except that at $Q^{2}=0$ we must have $\sigma_{\mathrm{L}}=0$ by gauge invariance. To satisfy this it is customary to put $R=Q^{2} / \mathrm{m}_{\rho}^{2}$, and some authors have used this in eq. (2). When that is done it leads to a big effect with a large cross section from the integral over $Q^{2}$. It is again known from electroproduction $[1,2]$ that $R$ does not rise at larger $Q^{2}$ and, apart from a possible excursion to $R \sim 1$ near $Q^{2} \lesssim 1 \mathrm{GeV}^{2}$, the data is consistent with $R \lesssim \frac{1}{4}$ at all $Q^{2}$. Consequently, $\sigma_{\mathrm{L}}$ cannot contribute much.

We have computed with two choices, meant to represent reasonable limits on the electroproduction data. One is $R=0$, i.e., no contributing from $\sigma_{\mathrm{L}}$. The other is a vector dominance $R$ which rises as $Q^{2}$ for small $Q^{2}$ and rises as $\ln Q^{2}$ at large $Q^{2}$,

$$
R=0.25\left[\left(1+0.36 / Q^{2}\right) \ln \left(1+0.36 / Q^{2}\right)-1\right],
$$

taken from ref. [12]. It seems unreasonable to us to get a larger effect from $R$, and this one only increases $\sigma$ by about $10 \%$ in general, so we believe that almost all the vector meson production should be via $\sigma_{\perp}$. Authors with larger $\sigma_{\mathrm{L}}$ will overestimate the production cross section.

There is also an interesting way some workers have found to considerably underestimate $\mathrm{d} \sigma / \mathrm{d} x \mathrm{~d} y$. Suppose one does not use the usual leptoproduction formalism, and writes an amplitude for fig. 1 with a propagator and a $\rho \mathrm{N}$ scattering amplitude for the lower vertex. Assuming this aplitude has a helicity dependence which is diagonal and helicity independent one finds a result as in eq. (2) but with only the $y^{2} \sigma_{\perp}$ term from the brackets. Since this is much the smallest term because most of the integral comes from small $y^{2}$, one obtains a very small estimate for $\mathrm{d} \sigma / \mathrm{d} x \mathrm{~d} y$.

Helicity independence at the $\rho$ pole, which is known to be rather good, requires $\sigma_{\mathrm{L}}=-\sigma_{\perp}$ there. These workers assume $\sigma_{\mathrm{L}}=-\sigma_{\perp}$ everywhere. This assumption violates gauge invariance which required $\sigma_{\mathrm{L}}=0$ at $Q^{2}=0$, as well as disagreeing with current data. 
In terms of the usual formalism this is the case where the hadron current is given by $\epsilon_{\mu}^{(\lambda)}(\rho) M(\rho \mathrm{N})=J_{\mu}$, the $\rho \mathrm{N}$ amplitude being just a function of $Q^{2}$ and $t$. Then $W_{\mu \nu} \propto \Sigma_{\lambda} \epsilon_{\mu}^{(\lambda)} \epsilon_{\nu}^{(\lambda)}$ gives only the $W_{1}$ term, and $W_{2}=0$ corresponds to $\sigma_{\perp}+\sigma_{\mathrm{L}}$ $=0$.

By applying the usual positivity arguments to the hadron tensor $W_{\mu \nu}$ one can show that $Q^{2} \sigma_{\mathrm{L}}$ is positive in both space-like and timelike regions so $\sigma_{\mathrm{L}}$ has a linear zero at $Q^{2}=0$, and care must be taken to use $\sigma_{L} \geqslant 0$ in the leptoproduction physical region.

For particles other than the $\rho$, production via the divergence of the current (e.g., the $\pi$ ) could contribute. In such cases $\sigma_{L}$ no longer must vanish at $Q^{2}=0$. However, such contributions are always proportional to the lepton mass squared in the cross section; even the $\pi$ exchange ones are not enhanced since $f_{\pi}^{2} /\left(M_{\pi}^{2}+Q^{2}\right)^{2} \propto$ $M_{\pi}^{2} /\left(M_{\pi}^{2}+Q^{2}\right)^{2}$ occurs. Consequently, we have neglected all such effects. If heavy leptons are produced diffractive production of pseudo scalars will be important and conversely.

\section{Kinematics}

Two aspects of the kinematics are important for the results, the limits of integration, and $t_{\min }$. We will present some details so it is clear what we have done, and to illustrate where the effects show up.

Basically, at the lower energies the kinematical limits suppress the cross section a lot, while at high energies the $t_{\mathrm{m} \text { in }}$ effect limits the cross section to small $x$. These
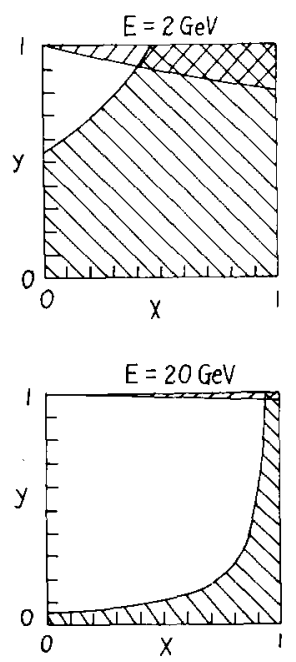

Fig. 3. Illustration of the kinematical limits, given by eq. (16) for the $x$ and $y$ integration. The shaded regions are excluded. 


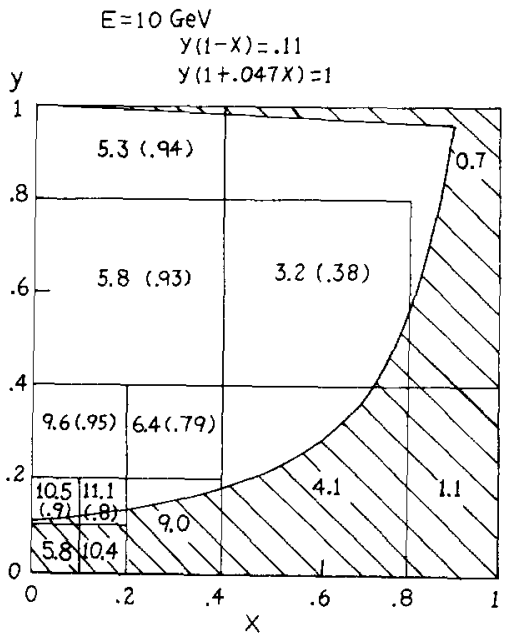

Fig. 4. Illustration of the kinematical limits and the behavior of the integrand in case (i). The number in each square represents the integrand without the $t_{\min }$ suppression. In the kinematically allowed region, the number inside each paranthesis represents $\mathrm{e}^{B t_{\mathrm{min}}}$.

effects are shown in fig. 3 and 4 where the kinematically allowed region is unshaded and at one energy the integrand and $\mathrm{e}^{B t}$ min values are shown. In this section we will speak in terms of $\rho$ production, but for the other states the replacement $m_{\rho}^{2} \rightarrow m_{\mathrm{V}}^{2}$ should always be made.

We have not found any simple approximate $t_{\min }$ which is reasonably accurate over the kinematical region of interest. It is not hard for numerical work to use the exact formula,

$$
\begin{aligned}
t_{\text {min }} & =\left(p-p^{\prime}\right)^{2}-\left(p_{0}-p_{0}^{\prime}\right)^{2} \\
& =\frac{m_{\mathrm{N}}^{2}}{2 W^{2}} \frac{\left(m_{\rho}^{2}+Q^{2}\right)^{2}}{\left(p_{0} p_{0}^{\prime}+p p^{\prime}+m_{\mathrm{N}}^{2}\right)}\left(\frac{p_{0}+p_{0}^{\prime}}{p+p^{\prime}}\right)^{2},
\end{aligned}
$$

where the invariant $t_{\min }$ has been evaluated in the hadron rest frame

$$
\begin{aligned}
& p_{0}=\left(W^{2}+m_{\mathrm{N}}^{2}-m_{\rho}^{2}\right) / 2 W, \\
& p=\left(p_{0}^{2}-m_{\mathrm{N}}^{2}\right)^{1 / 2}, \\
& p_{0}^{\prime}=\left(W^{2}+m_{\mathrm{N}}^{2}+Q^{2}\right) / 2 W, \\
& p^{\prime}=\left(p_{0}^{\prime 2}-m_{\mathrm{N}}^{2}\right)^{1 / 2} .
\end{aligned}
$$

The second form in eq. (13) is useful because it never requires a difference of two comparable numbers. 
When $W^{2} \gg Q^{2}, m_{\mathrm{N}}^{2}$ (Regge limit), we have approximately

$$
t_{\text {min }} \simeq-\frac{\left(m_{p}^{2}+Q^{2}\right)^{2}}{(2 E y)^{2}(1-x)^{2}},
$$

which illustrates that $\mathrm{e}^{B t}$ min suppresses $\mathrm{d} \sigma / \mathrm{d} x \mathrm{~d} y$ exponentially for large $Q^{2}$ and for $x \geq \frac{1}{2}$. Such an approximation requires $y \gg m_{\mathrm{N}} / 2 E$.

Next consider the integration limits. They are shown in fig 3 on an $x y$ plot. The limits are given by

$$
\begin{aligned}
& y\left(1+m_{\mathrm{N}} x / 2 E\right)=1, \\
& E y(1-x)=m_{\rho}+m_{\rho}^{2} / 2 m_{\mathrm{N}}, \\
& x=0,
\end{aligned}
$$

which can be derived, for example, by requiring the lepton scattering angles to have $\cos ^{2} \theta \leqslant 1$ and requiring $W^{2} \geqslant\left(m_{\rho}+m_{\mathrm{N}}\right)^{2}$. The lepton masses are assumed zero. The inequalities give the allowed region, bounded by the above lines. As the figures show, a good deal of the $x y$ plot is excluded at typical experimental energies. In particular, the region $y \leqslant\left(m_{\rho}+m_{\rho}^{2} / 2 m\right) / E(1-x)$ for small $x$ where the integrand is large must be kept excluded in any approximation scheme to avoid a significant overestimate.

\section{Results}

We have discussed the main interest of most of the results in the introduction. Here we simply present the numerical cross sections with a few comments.

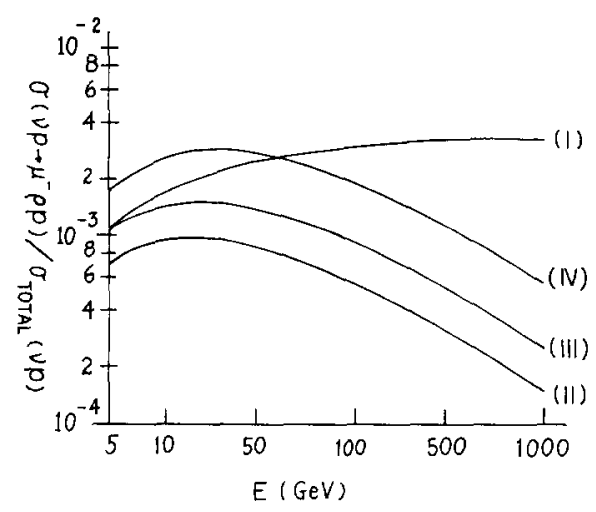

Fig. 5. The energy dependence of the relative $\rho^{+}$production rates for the four cases discussed in sect. 3. These results are obtained with $R=0$; those with $R$ given by eq. (12) are approximately 10 to $20 \%$ higher. $\sigma_{\text {tot }}(\nu \mathrm{p})$ is taken to be $0.7 E \times 10^{-38} \mathrm{~cm}^{2}$ with $E$ in $\mathrm{GeV}$. 


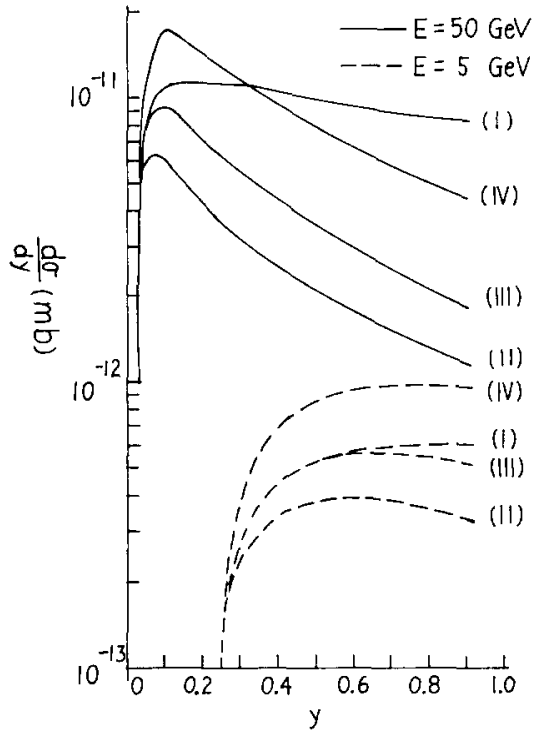

Fig. 6. The $y$ distribution for the $\rho^{+}$production for the four cases with $R=0$.

The Michigan, Fermilab, Hawaii, LBL collaboration has reported preliminary results for limits on $\rho^{+}, \mathrm{B}^{+}, \mathrm{A}_{1}^{+}$production [13] in $\nu \mathrm{p} \rightarrow \mu^{-} \mathrm{V}^{+} \mathrm{p}$. Their limits are consistent with our numbers and only exceed them by a little, so with the increased statistics available in the next few monts it is likely that a signal will be seen. Their limits are considerably below some of the estimates for $\rho$ and $A_{1}$ in ref. [8-10].

Fig. 5 shows the four cases for the $\rho$ described in sect. 3. The three that could correctly describe the data have significantly different $y$ distributions shown in fig. 6 , so a measurement of the $y$ distribution will nicely distinguish among the al-

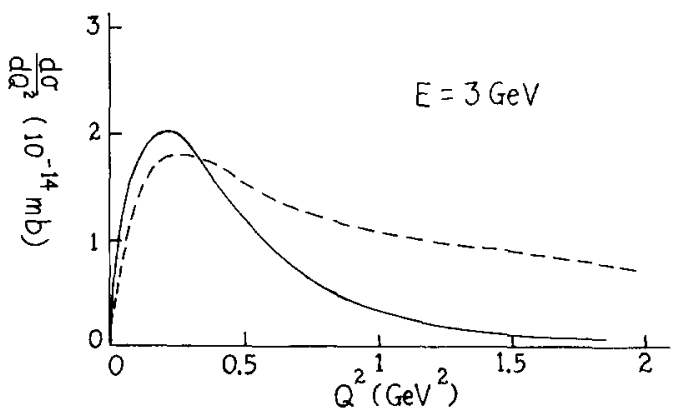

Fig. 7. The $Q^{2}$ distribution for the $\rho^{+}$production. The solid line represents the results obtained from case (i) of sect. 3 with $R$ given by eq. (12), whereas those with $R=0$ are about the same at small $Q^{2}$ and become $5 \%$ lower at $Q^{2}=1.5 \mathrm{GeV}^{2}$. The dashed line is reproduced from the calculation in ref. [9] for comparison. 


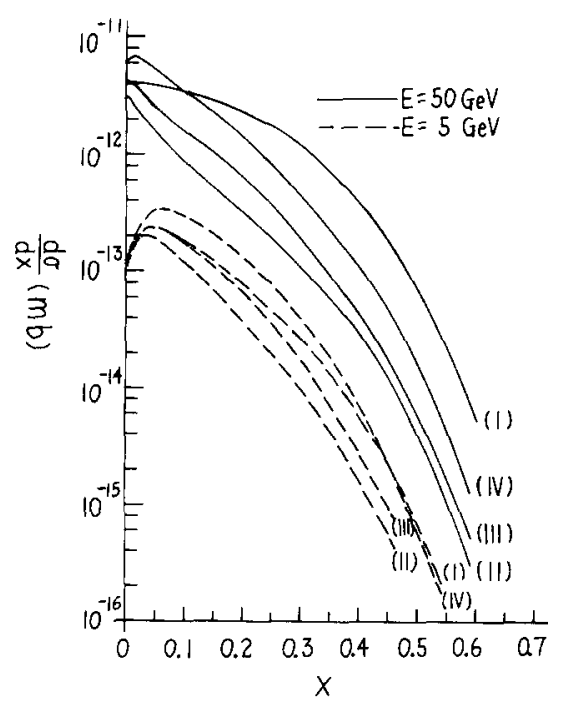

Fig. 8. The $x$ distribution of the $\rho^{+}$production for the four cases discussed in sect. 3 with $R=0$. These distributions vanish in the kinematical limits for very small values of $x$.

ternatives and the associated physics. The rates only differ a little in the 10-20 $\mathrm{GeV}$ region and each $y$ distribution gives a definite rate. Fig. 7 shows the $Q^{2}$ distribution for one alternative (case (i) of sect. 3) and a comparison curve from ref. [9] to show the more rapid fall-off in $Q^{2}$ required by the electroproduction data.

Fig. 8 shows $x$ distributions. They are determined by phase space and $t_{\min }$ effects more than the other distributions. Note however that the ratio of cases (i) and (iii) varies by an order of magnitude over a useful part of the range.

Fig. 9 shows the rates integrated over $x$ and $y$ for $\rho^{+}, \mathrm{B}^{+}, \mathrm{A}_{1}^{+}, \mathrm{F}^{*+}$. Two curves

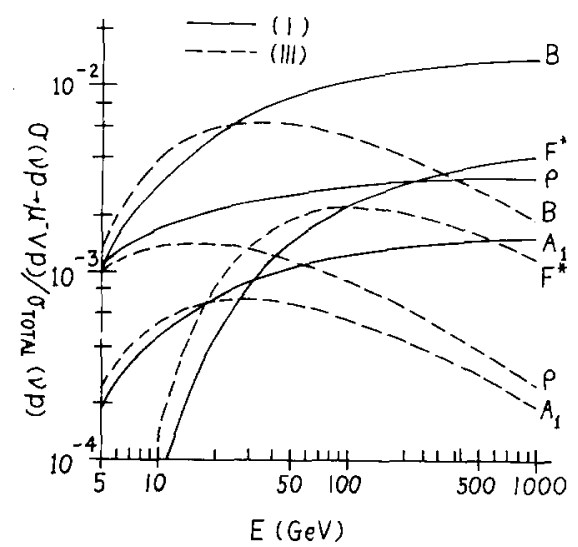

Fig. 9. The relative rates of the $\rho^{+}, \mathrm{B}^{+}, \mathrm{A}_{1}^{+}$, and $\mathrm{F}^{*+}$ production for cases (i) and (iii) with $R=0$. 
are shown for each, one from case (i) of sect. 3 and one from case (iii) of sect. 3. These two cases give the same small $Q^{2}$ behavior and differ by a power of $Q^{2}$ at large $Q^{2}$. Case (i) gives a divergent $Q^{2}$ integral so $\sigma\left(\nu \mathrm{p} \rightarrow \mu^{-} \mathrm{V}^{+} \mathrm{p}\right)$ grows as the first power of $E$ and $\sigma\left(\nu \mathrm{P} \rightarrow \mu^{-} \mathrm{V}^{+} \mathrm{p}\right) / \sigma(\nu \mathrm{p}) \rightarrow$ constant; this may be physically unrealistic even though it gives perhaps the best small $Q^{2}$ description, so it should be interpreted as an upper limit. Case (iii) with $\sigma_{\perp} \sim 1 / Q^{4}$ probably gives a lower limit to the rate. Whether the $1 / Q^{4}$ comes from a faster decrease of $\sigma_{\text {tot }}\left(\gamma_{V} \mathrm{p}\right)$ at larger $Q^{2}$ than at smaller $Q^{2}$, or from $\sigma_{\rho} / \sigma_{\text {tot }}$ vanishing at large $Q^{2}$, or from an intermediate vector boson propagator, is partly a matter of interpretation and does not directly enter. In any case, in the $10-25 \mathrm{GeV}$ region where the vector meson should first be detected the two limits only differ a little, and then a measurement at higher energies adds new physics information on the $Q^{2}$ dependence. This sensitivity to high $Q^{2}$ is an intrinsic aspect of the $\nu$ reactions because there is no propatagor at present $Q^{2}$ values. At $E=50 \mathrm{GeV}$ (say) there is a significant amount of $\sigma(\nu \mathrm{p} \rightarrow$ $\mu^{-} \rho^{+} \mathrm{p}$ ) coming from $Q^{2} \geqslant 20 \mathrm{GeV}^{2}$, so the results are not insensitive to $\mathrm{W}$ boson masses in the $50-100 \mathrm{GeV}$ region.

To conclude, we emphasize again (see introduction for details) the importance of these total rates. Confirming the $\rho$ prediction is of fundamental importance to weak interaction theory, as it is essentially a well defined translation of ep $\rightarrow e \rho^{0} \mathrm{p}$ into $\nu \mathrm{p} \rightarrow \mu^{-} \rho^{+} \mathrm{p}$. If the $\mathrm{A}_{1}$ is not present at the expected level it will confirm the evidence from hadron reactions that no $A_{1}$ exists, and confirm that a possibly basic puzzle exists for weak interaction theory and the quark model. Finding the $\mathrm{B}^{+}$ would confirm the existence of strong second class currents in particle physics and possibly provide a measurement of their strength, while the absence of the $\mathrm{B}^{+}$would be inconsistent with the second-class current model of ref. [6]. Detection of $\mathrm{F}^{*+}$ would be of considerable value in interpreting dilepton production in $\nu$ reactions and of considerable intrinsic interest. We find about $0.2 \%$ of $\sigma_{\mathrm{T}}(\nu \mathrm{p})$ for $\sigma\left(\nu \mathrm{p} \rightarrow \mu^{-} \mathrm{F}^{*+} \mathrm{p}\right)$. If $\sigma_{\mathrm{T}}\left(\mathrm{F}^{*} \mathrm{p}\right) \simeq 10 \mathrm{mb}$ from the additive quark model, and $\mathrm{F}^{*} \mathrm{p}$ scattering has a slope $B=5 \mathrm{GeV}^{-2}$, then $\sigma_{\mathrm{el}} \simeq \sigma_{\mathrm{T}}^{2} / 16 \pi \mathrm{B} \simeq 1 \mathrm{mb}$. So the total charm production via an $\mathrm{F}^{*}$ dominated current would be about 10 times our number or about $2 \%$. (If the axial vector $\mathrm{F}^{*}$ exists (though the $\mathrm{A}_{1}$ probably does not) this could be increased by a factor of about 2.) Then it must be multiplied by the muon or electron semi-leptonic branching ratio (say 10\%) to get the total dilepton rate, which would then be of or$\operatorname{der} 0.2 \%$. For $\bar{\nu}$ reactions $\sigma_{\mathrm{T}}$ is 3 times smaller while the $\mathrm{F}^{*}$ production is about the same, so this increases to a number of order $0.6 \%$. The corresponding neutral current reactions are discussed in the introduction; only $A_{1}^{0}$ is expected to be large in the conventional theory and if there is no $A_{1}$ that will be small too so there may be very little diffractive elastic neutral current cross section altogether.

\section{References}

[1] G. Wolf, Proc. 1975 Int. Symp. on lepton and photon interactions at high energies, SLAC, ed. W.T. Kirk (1975). 
[2] SLAC-Santa Cruz experiment reported by R.F. Mozley in Proc. 1975 Int. Symp. on lepton and photon interactions at high energies, SLAC, ed. W.T. Kirk.

[3] J.G. Morfin, Proc. 1975 Int. Symp. on lepton and photon interactions at high energies, SLAC, ed. W.T. Kirk.

[4] G.L. Kane, The $A_{1}$ problem and should SU (3) multiplets be complete?, New directions in hadron spectroscopy (Argonne National Laboratory, 1975). ed. S.L. Kramer and E.L. Berger,

[5] M.-S. Chen, G.L. Kane and J. Krisch, Phys. Rev. D13 (1976) 1499.

[6] M.-S. Chen, F.S. Henyey and G.L. Kane, A model for charged second-class currents, UM HE 76-18.

[7] S. Adler et al., Phys. Rev. D12 (1975) 3522.

[8] J. Pumplin and W. Repko, Phys. Rev. D12 (1975) 1376;

J.M. Wang and L.L. Wang, Phys. Letters 61 B (1976) 377;

B.A. Arbuzov, S.S. Gershtein and V.N. Folomeshkin, Phys. Letters 58B (1975) 64;

M.B. Einhorn and B.W. Lee, Phys. Rev. D13 (1976) 43;

V. Barger, T. Weiler and R.J.N. Phillips, Phys. Rev. D12 (1975) 2628.

[9] M.K. Gaillard, S.A. Jackson and D.V. Nanopoulous, Nucl. Phys. B102 (1976) 326.

[10] C.A. Piketty and L. Stodolsky, Nucl. Phys. B1 5 (1970) 571.

[11] B.P. Roe, Phys. Rev. Letters 21 (1968) 1666; 23 (1969) 692.

[12] R. Devenish and D. Schildknecht, DESY preprint 75/18, Off-diagonal generalized vector dominance: a comparison with recent ep deep inelastic data.

[13] C.T. Coffin et al., Michigan-Fermilab-Hawaii-LBL Collaboration preprint, submitted to 18th Int. Conf. on high-energy physics, July 1976, by B. Roe. 